\title{
AKZEPTANZ
}

\section{Neue Technologien erfolgreich einführen}

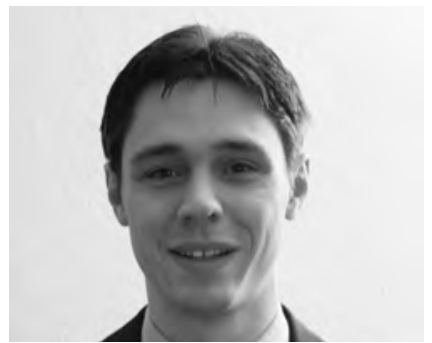

VON ROBERT LEHMANN Dr. Robert Lehmann ist Lehrkraft für besondere Aufgaben an der Katholischen Universität Eichstätt-Ingolstadt und dort für empirische Forschungsmethoden und E-Learning zuständig Er betreut in den Arbeitsstellen für Sozialinformatik und NPO-Controlling/SROI die Forschungsprojekte zu Fragen der Wirkungsmessung Sozialer Arbeit und Implementation neuer Technologien in Unternehmen der Sozialwirtschaft. Internet http://www.ku.de/swf

\author{
Neue Technologien in sozialwirtschaftlichen \\ Unternehmen stellen für Management und \\ Mitarbeitende immer eine Herausforderung dar. \\ Beachtet man jedoch einige Erkenntnisse der \\ Akzeptanz-Forschung, steigt die Chance, dass der \\ Implementationsprozess erfolgreich verläuft.
}

Inzwischen ist es auch in der Sozialwirtschaft Usus, dass innovative Technologien, allen voran fachspezifische IT-Lösungen, in den Unternehmen auf der Basis von strategischen Leitungsentscheidungen eingeführt werden. In den letzten Jahren mussten jedoch immer mehr Unternehmen die Erfahrung machen, dass die Implementation einer neuen Technologie bei ihnen an der mangelnden Bereitschaft der Mitarbeitenden scheiterte, diese zu nutzen. Diese Erfahrung ist nicht neu, die Forschung zur Akzeptanz technischer Innovationen geht bis in die 1970 er Jahre zurück. Ein weit verbreitetes Modell, das die Abläufe bei der Entwicklung von Akzeptanz einer neuen Technologie bei den Mitarbeitern erklärt, ist das Technology Acceptance Model (1).

Der Kern des Modells ist die Annahme, dass eine tatsächliche Nutzung stark, aber nicht ausschließlich, von der Nutzungseinstellung, also der geplanten Nutzung abhängt. Da die tatsächliche Nutzung kaum direkt beeinflusst werden kann, wurde in der Entwicklung des Modells ein großer Fokus auf die Faktoren gelegt, die auf die Nutzungseinstellung einwirken. Die beiden Hauptfaktoren bestehen zum einen in dem wahrgenommenen Nutzen bei den zukünftigen Anwendern. Hier ist insbesondere das Adjektiv "wahrgenommen" wichtig. Es ist also weniger der objektive Nutzen von Bedeutung, als die Einschätzung der Mitarbeitenden bezüglich des Nutzens der Anwendung. Der zweite Hauptfaktor ist die wahrgenommene Einfachheit der Bedienung. Auch hier ist wieder die subjektive Bewertung durch die Nutzer relevant, die sich oft eher an Gewohnheiten, als an objektiven Erkenntnissen der Usability-Forschung orientiert. In zahllosen Studien wurde dieses Modell um weitere Faktoren erweitert, die sich auf mindestens einen der beiden Hauptfaktoren auswirken.

Dabei sind auf der Seite des wahrgenommenen Nutzens insbesondere die Jobrelevanz und die Outputqualität der neuen IT-Technik hervorzuheben. Es ist durchaus entscheidend, inwiefern die Nutzer die Neuerung als relevant für ihren Arbeitsbereich erleben und welche Qualität sie den Ergebnissen zuschreiben. Auf der Seite der Bedienung bedürfen insbesondere die computerbezogene Selbstwirksamkeitserwartung und die Computerangst einer Erklärung. Die computerbezogene Selbstwirksamkeitserwartung setzt auf dem psychologischen Konzept der allgemeinen Selbstwirksamkeitserwartung nach Bandura auf. Es beschreibt die Erwartungshaltung eines Menschen, eine gestellte Aufgabe mit Hilfe der eigenen Fähigkeiten bewältigen zu können. Im hier dargestellten Modell wird das auf die Erwartungshaltung, computerbezogene Aufgaben bewältigen zu können, angewandt. $(2,3)$

Das Konzept der Computerangst beschreibt eine irrationale Angst, durch 
die unsachgemäße Benutzung von Computern großen, irreparablen Schaden zu verursachen. Auch wenn es zunächst so scheint, als handele es sich hier um ein Problem, das vor allem ältere Menschen mit wenig IT-Erfahrungen betrifft, ist Computerangst in geringer Ausprägung auch bei jüngeren Altersschichten anzutreffen. (4)

Wie kann diese Theorie nun aktuelle Implementationsprozesse in der Sozialwirtschaft unterstützen?

\section{Fallbeispiel: So kann es gehen}

Fall 1: Hier wurde eine virtuelle Lernumgebung zu einem E-Mail Programm entwickelt und in zwei unterschiedlichen Szenarien angewandt. Im einen Szenario handelte es sich um Mitarbeitende eines Jugendamts, die mit dem E-Mail Programm täglich arbeiteten und an deren Fragen und Problemen die Inhalte orientiert waren. Das andere Szenario waren Studierende der Sozialen Arbeit, die die Lernumgebung im Rahmen eines Pflichtseminars besuchen mussten. Sie wurden jedoch darüber informiert, dass es sich um authentisches Material aus ihrem zukünftigen Berufsleben handelt. Beide Gruppen wurden nach der Bearbeitung der Lernumgebung zu ihrer Akzeptanz und zu ihrer Nutzungseinstellung zur Lernumgebung befragt. Diese war auf der Seite der Jugendamtsmitarbeiter signifikant positiver ausgeprägt als bei den Studierenden. Einerseits überrascht dieses Ergebnis angesichts des deutlich niedrigeren Altersdurchschnitts der Studierenden und allgemeinen Annahmen der Technikaffinität und Lernbegeisterung dieser Gruppe. Berücksichtigt man jedoch das Technology Acceptance Model, wird deutlich, dass sich die Gruppen aufgrund der unterschiedlichen Settings, in denen sie lernten, deutlich im Bezug auf die Freiwilligkeit und die Jobrelevanz der verwendeten Technologie unterschieden und die diesbezüglichen Voraussetzungen bei den Studierenden deutlich schlechter waren. Diese Unterschiede begründeten dann auch einen deutlich schlechteren Lernerfolg bei den Studierenden. (5)

Fall 2: Der Geschäftsführer eines kleinen gemeinnützigen Trägers der Sozialen Arbeit in Oberbayern wollte die Website des Vereins inhaltlich dynamischer gestalten. Ziel war ein Content Management System zu implementieren, das es unterschiedlichen Bereichen des Vereins möglich machen sollte, ihren Webauftritt innerhalb der Gesamtwebsite zu pflegen und möglichst häufig mit aktuellen Informationen zu versehen. Bisher gab es einen externen Dienstleister, der in großen zeitlichen Abständen die Website um neue Informationen ergänzte. Neben der Auswahl einer geeigneten technischen Lösung war von Beginn an entscheidend, dass ein großer Teil des Mitarbeiterstamms bereit ist, die neue Technologie zu nutzen. Um eine positive Nutzungseinstellung zu generieren, musste von Anfang deutlich werden, welche Relevanz die Pflege der Website für die einzelnen Fachbereiche hat (Jobrelevanz) und dass die Ergebnisse, die mit dem Content Management System erzielt werden, hohe Qualität haben (Outputqualität). Ein Content Management System erfordert von den Personen, die damit arbeiten zwar keine Programmierkenntnisse, dennoch ist die Bedienung nicht selbsterklärend. Um sicherzustellen, dass die Bedienung als einfach wahrgenommen wird, wurde frühzeitig darauf geachtet, dass die zukünftigen Nutzer über eine praxisnahe Einführung eine entsprechende Selbstwirksamkeitserwartung aufbauen und Gelegenheit bekommen, eventuelle Computerängste abzubauen. So konnte erreicht werden, dass aus allen Geschäftsbereichen ausreichend Mitarbeitende die Verantwortung für ihren Teil der Website übernehmen und ein lebendiges Kommunikationsinstrument geschaffen werden.

\section{Resümee}

Für die praktische Arbeit und Implementation von neuen Technologien bieten Modelle wie das Technology Acceptance Model die Gelegenheit, systematisch relevante Einflussfaktoren auf die Akzeptanz zu identifizieren und Maßnahmen zu konzipieren, die an den Faktoren ansetzen, die im eigenen Unternehmen am relevantesten erscheinen. So kann erreicht werden, dass die Anwender frühzeitig eine positive Nutzungseinstellung aufbauen und die neue Technologie den geplanten Nutzen bringt.

\section{Anmerkungen}

(1) V. Venkatesh und H. Bala, Technology Acceptance Model 3 and a
Research Agenda on Interventions, Decision Sciences, Bd. 39, Nr. 2, S. 273-315, 2008.

(2) A. Bandura, Self-efficacy: Toward a unifying theory of behavioral change, Psychological Review, Bd. 84, Nr. 2, S. 191-215, 1977.

(3) D. Compeau und C. Higgins, Computer Self Efficacy: Development of a Measure and Initial Test, Management Information Systems Quarterly, Bd. 19, Nr. 2, S. 189-211, 1995.

(4) G. A. Marcoulides, C. Emrich, und L. D. Marcoulides, Testing for Multigroup Invariance of the Computer Anxiety Scale, Educational and Psychological Measurement, Bd. 68, Nr. 2, S. 325-334, 2008.

(5) R. Lehmann, Lernstile als Grundlage adaptiver Lernsysteme in der Softwareschulung, Bd. 54. Münster, München [u. a.]: Waxmann, 2010.

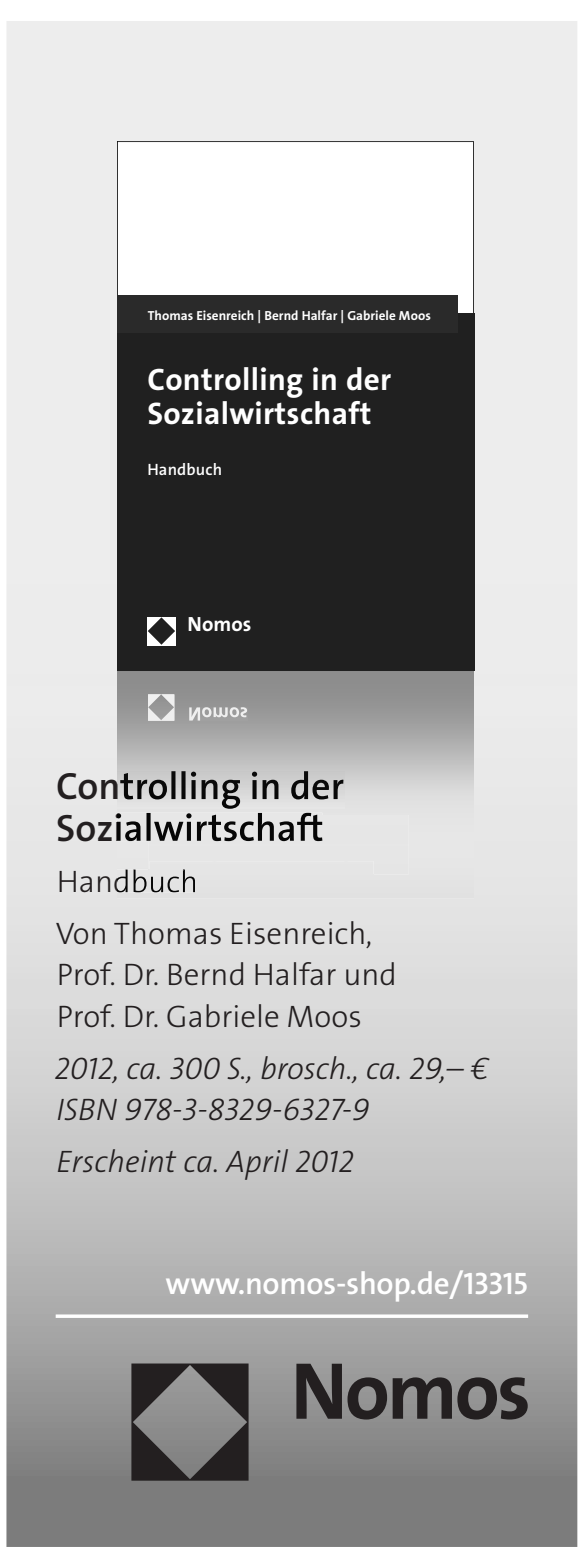

